\title{
Constraining Supernova Progenitors
}

\author{
Hanindyo Kuncarayakti* \\ ${ }^{1}$ Millennium Institute of Astrophysics (MAS), Nuncio Monseñor Sótero Sanz 100, Providencia, \\ Santiago de Chile 7500011 \\ ${ }^{2}$ Departamento de Astronomía, Universidad de Chile, Casilla 36-D, Santiago de Chile \\ E-mail: haninedas.uchile.cl
}

\begin{abstract}
There have been a large number of supernovae observed. The progenitors of these explosions are thought to be evolved stars, although still many details of the events and precursor objects are not very well understood. In the recent decades there have been a number of detections of supernova progenitor stars in pre-explosion Hubble Space Telescope archival images, which helped to firmly identify and characterize the physical properties of the exploded star. In addition to archival direct detections, environment studies have also been providing important clues on the nature of the supernova progenitors. Although mass and metallicity have been regarded as the main parameters governing stellar evolution, binarity has now been gaining more support as another important factor in the production of hydrogen-poor core-collapse supernovae.
\end{abstract}

XI Multifrequency Behaviour of High Energy Cosmic Sources Workshop -MULTIF15-, 25-30 May 2015

Palermo, Italy

${ }^{*}$ Speaker. 


\section{Introduction}

Supernovae ( $\mathrm{SNe}$ ) are observed as the appearance of a new star in the sky, with luminosity rivaling the host galaxy where it resides. Some of these events occurred within the Milky Way galaxy and have been witnessed and reported by observers since at least $\sim 2000$ years ago (see e.g. Aschenbach, 2015). The current era of astronomy has been witnessing exponentially increasing rate of SN discoveries currently in the order of several SNe per day (Gal-Yam et al., 2013), mainly driven by modern surveys utilizing wide-field CCD cameras and robotized telescopes. Despite this large number of events, there is still limited knowledge on the physical characteristics and evolutionary status of the SN progenitor stars. Traditionally, SNe are divided into several subclasses according to the optical spectrum near maximum light (see Filippenko, 1997, for a review). TypeII SNe, which show strong hydrogen lines in their spectra, are thought to be massive stars still retaining their outer hydrogen envelopes at the time of the explosion. On the other hand, type-I SNe which are hydrogen-poor are thought to be composed of two different populations of evolved stars. SNe type-Ib, whose spectra show prominent He lines, and type-Ic, which are depleted of both $\mathrm{H}$ and $\mathrm{He}$, are thought to be the explosions of stripped massive stars not unlike Wolf-Rayet (WR) stars, whereas type-Ia SNe are completely different compared to the other SN subtypes i.e. they are not produced from the explosion following core collapse of a massive star but instead from a white dwarf progenitor undergoing thermonuclear explosion (see e.g. Lipunov, 2015).

There have been a significant number of theoretical works predicting massive star evolution and the subsequent supernova. Initail mass and metallicity of the progenitor star is often regarded as the main physical parameters governing stellar evolution. Stellar evolution calculations such as those presented by Heger et al. (2003) and Georgy et al. (2009) describe the mapping of which stars in the mass-metallicity plane will produce a particular SN type and compact remnant, but these kinds of predictions still have to be verified by observational data. During the recent decades, there have been new breakthroughs in the effort of constraining the different progenitor stars of $\mathrm{SNe}$. These include direct detections of the progenitor stars in pre-explosion archival images and statistical studies of the SN environments, which will be discussed in the following sections.

\section{Direct detections of supernova progenitors}

One powerful way to constrain the SN progenitors is by identifying the precursor star in images obtained before the explosion. The Hubble Space Telescope (HST) has been providing a unique opportunity to resolve nearby SN sites and identify the progenitor star, owing to its capability of obtaining high-resolution images unaffected by the earth's atmosphere. Prior to HST, SN progenitor stars have been identified only for two very nearby SNe: 1987A (White \& Malin, 1987) in LMC (50 kpc) and 1993J (Aldering et al., 1994) in M81 (3.6 Mpc).

In order to accurately identify the progenitor object in the archival images, precise astrometry of the SN needs to be obtained to see where the SN falls in the pre-SN images. This is often achieved also using HST or ground-based adaptive-optics observations of the SN while it is still present. With this technique, the SN position could be determined with miliarcsecond precision and thus enabling the association of the $\mathrm{SN}$ with a progenitor candidate identified in pre-SN images. Photometry of the progenitor candidate then places the star on the Hertzprung-Russell Diagram. 
The position of the star later could be compared with stellar evolutionary tracks of various initial masses. Thus, the evolutionary status and initial mass of the progenitor star candidate could be estimated with confidence.

While powerful, ideally this direct detection technique should be accompanied by the confirmation of the disappearance of the progenitor candidate at late times to ascertain the correct progenitor identification. Excellent examples of this procedure exist only for a handful of $\mathrm{SNe}$ including SNe 1987A, 1993J, and more recently 2011dh (Folatelli et al., 2014). In Figure 1, the sequence of HST images showing the SN field before and after the explosion reveals the identification of the progenitor star candidate and its disappearance, leaving behind a blue binary companion star as predicted by the models calculations, which unambiguously confirms the star as the progenitor of the SN. In most of the cases, progenitor candidate disappearance is unconfirmed, therefore there is always the danger of false identification in which the assumed progenitor candidate still present long after the SN explosion (e.g. Maund et al., 2015).

Smartt et al. (2009) and Eldridge et al. (2013) summarized the efforts to identify nearby SN progenitors using the HST within $28 \mathrm{Mpc}$. It was found that type-IIP SNe, which are the most common explosion by volume, are produced by red supergiant stars. These stars still retain their extended hydrogen envelope at the time of explosion and belong to the $\sim 8-20 \mathrm{M}_{\odot}$ initial mass range. On the other hand, the search for progenitors of type-I SNe has been met with non-detections, except one possible case of SN Ib iPTF13bvn. This raises the question whether the supposed progenitors of $\mathrm{SNe} \mathrm{Ib} / \mathrm{c}$ are really bright massive stars similar to the classical WR stars. WR stars are initially massive $\left(\mathrm{M}_{Z A M S} \gtrsim 25 \mathrm{M}_{\odot}\right)$ and lost their hydrogen envelope via strong, metallicity-driven stellar winds. Alternatively, it has been argued that possibly some Ib/c progenitors could have been sub WR-mass stars in a close binary system. Other explanations of these non-detections include the possibility that pre-SN WR stars are optically faint (Yoon et al., 2012), and the majority of massive stars over $\sim 18 \mathrm{M}_{\odot}$ form black holes quietly without displaying visible SN (Smartt, 2015).

\section{Statistical studies of supernova environments}

Another powerful method to constrain the SN progenitors is to study the local environments. This can be done in a statistical way, as one can increase the sample size easily by re-observing the SN sites after the SN disappeared to obtain necessary information of the stellar populations hosting the SNe. It has been long recognized that type-II and Ib/c SNe occur in predominantly star-forming host galaxies, while type-Ia SNe occur in both passive and star-forming hosts. This immediately suggests that the progenitors of $\mathrm{SNe}$ II and Ib/c are associated with young stellar populations containing massive, ionizing stars. Since core-collapse SNe are thought to be produced by young, massive stars, it only logical to study their association to the star-forming region from which they emerge. As massive stars evolve quickly (in the order of $\sim \mathrm{Myr}$ ), they should not have moved far from their birthplaces. Using pixel statistics technique applied to the SN host galaxies, Anderson et al. (2012) showed that SNe Ic tend to have closer association with star-forming regions compared to the other SN subtypes. Following SNe Ic are consecutively SNe Ib, II, and Ia. This was interpreted as the sequence of decreasing progenitor mass for each SN subclasses. This result confirmed the notion that there is a significant fraction of type $\mathrm{Ib} / \mathrm{c} \mathrm{SNe}$ that are produced by stars more massive than SN II progenitors. As has been shown by Leloudas et al. (2011) who derived 


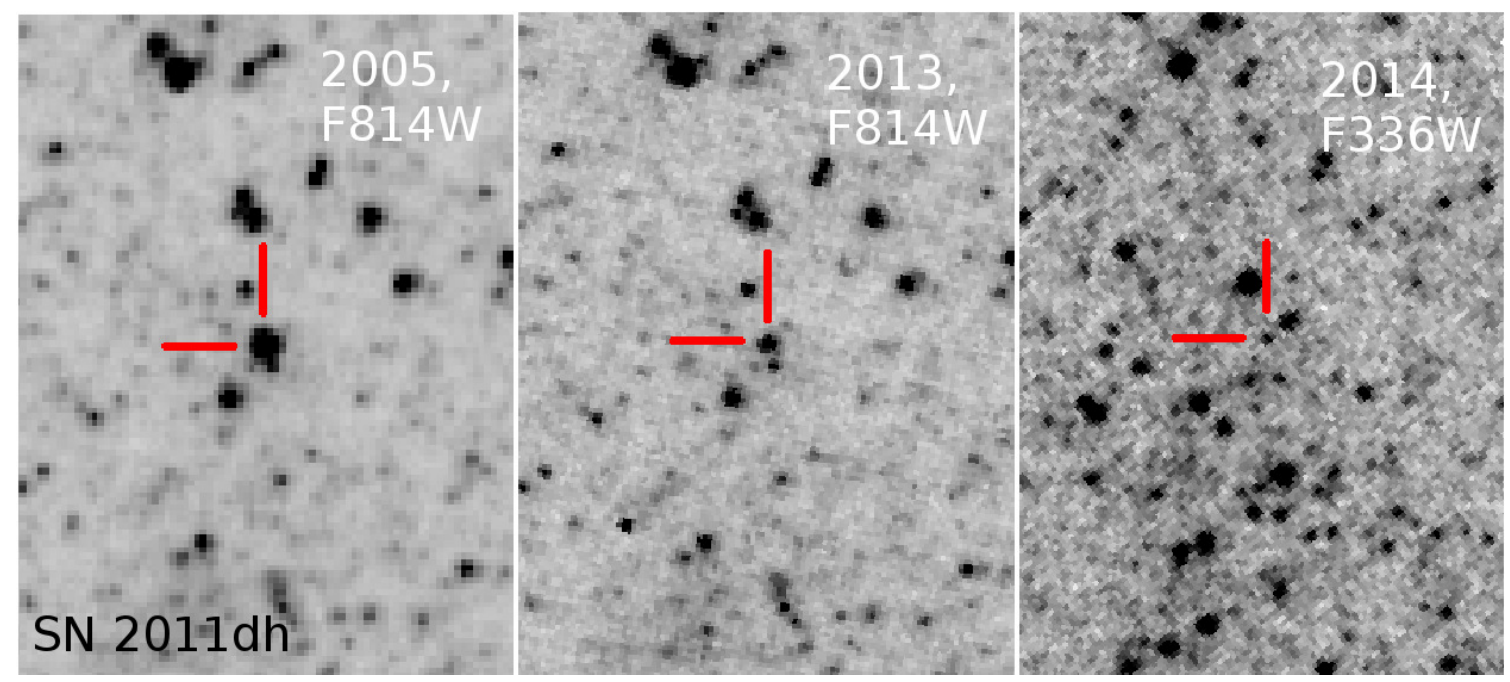

Figure 1: HST images of SN 2011dh; north is up and east is to the left. Left: ACS/WFC-F814W (2005), showing the pre-SN progenitor star as indicated by the red crosshairs. Center: WFC3/UVIS-F814W (2013), showing the fading SN that is now fainter than the progenitor star. Right: WFC3/UVIS-F336W (2014), showing the remaining blue binary companion.

the ages of $\mathrm{SN} \mathrm{Ib/c} \mathrm{explosion} \mathrm{sites} \mathrm{by} \mathrm{slit} \mathrm{spectroscopy} \mathrm{of} \mathrm{the} \mathrm{underlying} \mathrm{H}$ II regions, there could be a mix of both young, massive ( $\gtrsim 25-30 \mathrm{M}_{\odot}$ ) and older, less massive progenitors of SNe $\mathrm{Ib} / \mathrm{c}$.

The environment of the SN host galaxy itself can also provide additional clues about the SN progenitor stars. Anderson et al. (2015) summarized the recent efforts in statistical studies of SN environments, in which they also presented new updated results. In that review, the host galaxies of the $\mathrm{SNe}$ are compared and it was found that in disturbed galaxies $\mathrm{SNe} \mathrm{Ib} / \mathrm{c}$ are more concentrated towards the galaxy center compared to SNe II. The same trend was observed also in normal (undisturbed) host galaxies, although not as strong. Disturbed galaxies are thought to possess a more uniform metallicity distribution throughout the galactic disk, i.e. having weaker metallicity gradient compared to the normal ones. The progenitors of $\mathrm{SN} \mathrm{Ib/c}$ are expected to have higher metallicity compared to SN II, due to the increasing power to remove the hydrogen envelope with higher metallicity via stellar wind mechanism. Therefore, one would expect to observe central preference of $\mathrm{SNe} \mathrm{Ib} / \mathrm{c}$ in undisturbed galaxies which are showing strong metallicity gradient, in comparison to the disturbed ones. However, the empirical result presented in Anderson et al. (2015) shows the contrary. This might be indicating that there is no or weak effect of metallicity in the differentiation of SNe II and Ib/c.

In Kuncarayakti et al. (2013a,b), integral field spectroscopy was used to study the immediate environments of nearby SNe, within a few tens of pc around the SN explosion site. This technique enables simultaneous collection of both spatial and spectral information of the parent stellar population of the $\mathrm{SNe}$, therefore increasing the observation efficiency and minimizing contamination from other unrelated stellar populations in deriving the SN progenitor's properties. Comparing with simple stellar population (SSP) models, the parent stellar populations of the SN were characterized and assuming coevality, the physical properties of the SN progenitors were derived. In that study, an indication was found that SN Ic progenitors are the most massive and metal-rich compared to 
the other SN subtypes. Some Ib/c SNe were also revealed to be associated with stellar populations significantly older than the lifetime of stars with mass $\gtrsim 25 \mathrm{M}_{\odot}$, which signifies that WR stars could not have been the progenitors of those SNe. This leads to the conclusion that some $\mathrm{SNe} \mathrm{Ib} / \mathrm{c}$ are produced by stars not massive enough to remove their hydrogen envelopes on their own via stellar wind. Such progenitor stars are thought to be massive stars $\lesssim 25 \mathrm{M}_{\odot}$ inside a close binary system which get rid of their hydrogen via Roche-lobe overflow in binary interactions. This again supports the idea that the progenitors of $\mathrm{SNe} \mathrm{Ib} / \mathrm{c}$ cannot be composed of single, initially massive WR stars only. Other studies using different methods such as initial mass function calculations (Smith et al., 2011) and nebular spectroscopy (Kuncarayakti et al., 2015) also suggest that there should be a significant fraction of binary stars contributing to the $\mathrm{SN} \mathrm{Ib/c} \mathrm{progenitor} \mathrm{population.}$

\section{Summary}

In contrast to the large number of SNe that have been observed, there are only a few SN progenitors that have been characterized. Direct progenitor detections in pre-SN archival images has proven to be a powerful method in constraining the progenitor stars. In addition, environment studies of SNe provides invaluable information on the statistical differences between the progenitors of $\mathrm{SNe}$ of different types. The combination of both approaches needs to be pursued in order to further our understanding of $\mathrm{SNe}$ and their progenitor stars. It is now generally accepted that most $\mathrm{SNe}$ type-II result from the explosions of red supergiant stars between $\sim 8-20 \mathrm{M}_{\odot}$, while for type-Ib and Ic SNe it is necessary to have the contributions from both WR stars and interacting massive binaries for the progenitors.

\section{Acknowledgments}

Support for HK is provided by the Ministry of Economy, Development, and Tourism's Millennium Science Initiative through grant IC120009, awarded to The Millennium Institute of Astrophysics, MAS. HK acknowledges support by CONICYT through FONDECYT grant 3140563.

\section{References}

Aldering, G., Humphreys, R. M., \& Richmond, M. 1994, SN 1993J: The optical properties of its progenitor, $A J, 107,662$

Anderson, J. P., Habergham, S. M., James, P. A., \& Hamuy, M. 2012, Progenitor mass constraints for core-collapse supernovae from correlations with host galaxy star formation, MNRAS, 424, 1372 [arXiv:1205.3802]

Anderson, J. P., James, P. A., Habergham, S. M., Galbany, L., \& Kuncarayakti, H. 2015, Statistical Studies of Supernova Environments, PASA, 32, e19 [arXiv:1504.04043]

Aschenbach, B. 2015, Age and Distance of Puppis A Revised, this volume

Eldridge, J. J., Fraser, M., Smartt, S. J., Maund, J. R., \& Crockett, R. M. 2013, The death of massive stars - II. Observational constraints on the progenitors of Type Ibc supernovae, MNRAS, 436, 774 [arXiv:1301.1975] 
Filippenko, A. V. 1997, Optical Spectra of Supernovae, ARAA, 35, 309

Folatelli, G., Bersten, M. C., Benvenuto, O. G., et al. 2014, A Blue Point Source at the Location of Supernova 2011dh, ApJL, 793, L22 [arXiv:1409.0700]

Gal-Yam, A., Mazzali, P. A., Manulis, I., \& Bishop, D. 2013, Supernova Discoveries 2010-2011: Statistics and Trends, PASP, 125, 749 [arXiv:1103.5165]

Georgy, C., Meynet, G., Walder, R., Folini, D., \& Maeder, A. 2009, The different progenitors of type Ib, Ic SNe, and of GRB, A\&A, 502, 611 [arXiv:0906.2284]

Heger, A., Fryer, C. L., Woosley, S. E., Langer, N., \& Hartmann, D. H. 2003, How Massive Single Stars End Their Life, ApJ, 591, 288 [arXiv:0212469]

Kuncarayakti, H., Doi, M., Aldering, G., et al. 2013a, Integral Field Spectroscopy of Supernova Explosion Sites: Constraining the Mass and Metallicity of the Progenitors. I. Type Ib and Ic Supernovae, AJ, 146, 30 [arXiv:1305.1105]

Kuncarayakti, H., Doi, M., Aldering, G., et al. 2013b, Integral Field Spectroscopy of Supernova Explosion Sites: Constraining the Mass and Metallicity of the Progenitors. II. Type II-P and II-L Supernovae, AJ, 146, 31 [arXiv:1306.2106]

Kuncarayakti, H., Maeda, K., Bersten, M. C., et al. 2015, Nebular phase observations of the TypeIb supernova iPTF13bvn favour a binary progenitor, A\&A, 579, A95 [arXiv:1504.01473]

Leloudas, G., Gallazzi, A., Sollerman, J., et al. 2011, The properties of SN Ib/c locations, A\&A, 530, A95 [arXiv:1102.2249]

Lipunov, V. M. 2015, The Mechanism of Supernova Ia Explosion, this volume

Maund, J. R., Fraser, M., Reilly, E., Ergon, M., \& Mattila, S. 2015, Whatever happened to the progenitors of supernovae 2008cn, 2009kr and 2009md?, MNRAS, 447, 3207

Smartt, S. J., Eldridge, J. J., Crockett, R. M., \& Maund, J. R. 2009, The death of massive stars - I. Observational constraints on the progenitors of Type II-P supernovae, MNRAS, 395, 1409 [arXiv:0809.0403]

Smartt, S. J. 2009, Progenitors of Core-Collapse Supernovae, ARAA, 47, 63 [arXiv:0908.0700]

Smartt, S. J. 2015, Observational Constraints on the Progenitors of Core-Collapse Supernovae: The Case for Missing High-Mass Stars, PASA, 32, e16 [arXiv:1504.02635]

Smith, N., Li, W., Filippenko, A. V., \& Chornock, R. 2011, Observed fractions of core-collapse supernova types and initial masses of their single and binary progenitor stars, MNRAS, 412, 1522 [arXiv:1006.3899]

White, G. L., \& Malin, D. F. 1987, Possible binary star progenitor for SN1987A, Nature, 327, 36

Yoon, S.-C., Gräfener, G., Vink, J. S., Kozyreva, A., \& Izzard, R. G. 2012, On the nature and detectability of Type Ib/c supernova progenitors, A\&A, 544, L11 [arXiv:1207.3683] 